\title{
Compressible and Choked Flows in Rotating Passages
}

\author{
Nolan J. Dyck, Anthony G. Straatman \\ The Department of Mechanical and Materials Engineering, Western University, London, Canada \\ Email:ndyck@uwo.ca
}

How to cite this paper: Dyck, N.J. and Straatman, A.G. (2019) Compressible and Choked Flows in Rotating Passages. Open Journal of Fluid Dynamics, 9, 1-21. https://doi.org/10.4236/ojfd.2019.91001

Received: October 19, 2018

Accepted: February 12, 2019

Published: February 15, 2019

Copyright $\odot 2019$ by author(s) and Scientific Research Publishing Inc. This work is licensed under the Creative Commons Attribution International License (CC BY 4.0).

http://creativecommons.org/licenses/by/4.0/

\begin{abstract}
The present study revisits the rotating duct problem examined by Polihronov and Straatman (J. Polihronov and A. G. Straatman, Phys. Rev. Lett. v. 109, p. 054504 (2012)). Starting from the general compressible Euler equations in a non-stationary reference frame closed form expressions for velocity, temperature, density and pressure along the duct are determined. The present results are more general than those obtained by Polihronov and Straatman, as the change of in-frame kinetic energy has been retained. The improvement of the present results over Polihronov and Straatman's is demonstrated by comparison with the results of a computational fluid dynamics study. The new results have been further generalized to the case of a rotating duct with varying cross-sectional area, and again for a general curved passage in three-dimensional space. The work required or derived from the rotating duct has also been computed. The choked flow condition within the passage of varying cross-sectional area has been identified, along with the constraints which must be placed on the Mach, Rossby, and tip Mach numbers to avoid choked flow. Finally, a straightforward technique to identify any locations where an ideal rotating flow in a constrained passage will become sonic has been presented.
\end{abstract}

\section{Keywords}

Compressible Flows, Rotational Flows

\section{Introduction}

In two recent publications Polihronov and Straatman [1] [2] have applied heuristic techniques to examine the energetics of confined fluid flow in a rotating reference frame. These works were completed in an effort to shed new light on the temperature separation phenomenon within the Ranque-Hilshe Vortex Tube 
(RHVT), first discovered by Ranque [3]. Presently, the literature contains no widely accepted explanation of the temperature separation phenomenon as noted in a recent review by Thakare et al. [4], but a fundamental understanding of rotating compressible flows appears to be a promising starting point.

Studies of rotating flows may be divided into two broad categories: flows through rotating passages, and swirling flows. Both types of flows share similar features, but the latter comes with increased complexity. We emphasize that the present work focuses on flows through rotating passages, and will tackle swirling flows in future publications.

Rotating incompressible flows in confined passages have been studied extensively, both analytically and numerically. An initial treatment of rotating flows has been provided by Greenspan [5], and later textbooks have offered additional perspectives [6] [7] [8]. More recent work has focused on two and three-dimensional flow within rotating passages. Tatro and Mollo-Christensen [9] have studied the Ekman layers at low Rossby number flows experimentally, noting the presence of type I and type II instabilities. Kristofferson and Andersson [10] have employed direct numerical simulations to study turbulent boundary layer flows inside rotating passages, finding the variation in mean velocity profiles with changes in Rossby number. Khesghi and Scriven [11] have used the finite element method to study rotating flows when neither the Ekman nor the Rossby numbers may be neglected, and revealed the presence of an inviscid core flow near the axis of the straight passage.

Outside of the publications by Polihronov and Straatman, rotating compressible flows in confined passages have received attention from a variety of research fields. Most notably, Seymour Lieblein submitted a NACA Technical Note in 1952 [12] wherein he developed a set of equations describing compressible flow in radial compressor blade passages, including a discussion of supersonic flow and the effects of losses. In later publications, it has become popular to define the rothalpy of a compressible fluid undergoing radial motion, wherein the rothalpy has been shown to be constant when the flow may be considered adiabatic and frictionless [13] [14]. Bosman [15] later showed that, for "all engineering intents and purposes", the error associated with the constant rothalpy assumption may be neglected. Discussions of rothalpy now appear in graduate level fluid mechanics texts such as Refs. [7] [8].

The objective of the present work is to re-analyze the rotating duct problem studied by Polihronov and Straatman, starting instead from the governing equations of fluid mechanics. We will systematically obtain closed form mathematical expressions for the density, temperature, pressure, and velocity profiles within rotating, one-dimensional, straight and curved passages with constant and spatially varying cross-sectional areas, under the assumption that the flow is compressible, adiabatic, and inviscid. The motivation for this work is to gain insight from the solutions about the mechanism responsible for the temperature separation phenomenon in the RHVT. 


\subsection{Governing Equations}

The conservation equations of mass, momentum, and energy have been appropriately transformed into a general, non-inertial reference frame by Combrinck and Dala [16] by applying the Galilean transformation technique to the stationary conservation equations as suggested by Kageyama and Hyodo [17]. Here we work only with the steady forms of these equations. The conservation of mass is

$$
\nabla \cdot(\rho \hat{\boldsymbol{u}})=0,
$$

where $\hat{\boldsymbol{u}}$ is the velocity in the rotating and accelerating reference frame, $\rho$ is the density, and $\nabla$ is the gradient operator. The inviscid, steady, conservation of momentum equation in a non-accelerating rotating frame in the absence of body forces is

$$
(\hat{\boldsymbol{u}} \cdot \nabla) \hat{\boldsymbol{u}}=-\frac{\nabla p}{\rho}+\underbrace{2 \hat{\boldsymbol{c}} \times \boldsymbol{\Omega}}_{\begin{array}{c}
\text { Coriolisi } \\
\text { acceleration }
\end{array}} \underset{\begin{array}{c}
\text { centrifiugal } \\
\text { acceleration }
\end{array}}{-(\hat{\boldsymbol{x}} \times \boldsymbol{\Omega}) \boldsymbol{\Omega}},
$$

where $p$ is the thermodynamic pressure, $\boldsymbol{\Omega}$ is the angular velocity of the frame (which can be unsteady in general), and $\hat{\boldsymbol{x}}$ is the position vector. $\hat{\boldsymbol{x}}$ is defined relative to the origin of a co-ordinate system about which rotation occurs. When heat conduction and external heat sources may be neglected, the conservation of internal energy is

$$
\rho \hat{\boldsymbol{u}} \cdot \nabla \varepsilon=-p(\nabla \cdot \hat{\boldsymbol{u}}),
$$

where $\varepsilon$ is the specific internal energy. Notice only the velocity vector $\hat{\boldsymbol{u}}$ and the position vector $\hat{\boldsymbol{x}}$ have been assigned the ${ }^{\wedge}$ symbol. This emphasizes that these quantities are transformed versions of their stationary frame counterparts. All other quantities under consideration are scalars, which are not affected by the transformation into the rotating frame, so the distinction between scalar quantities in the rotating frame and their counterparts in the stationary frame is not made.

\subsection{Auxillary Equations}

All fluids analyzed in this work are characterized by the ideal gas equation of state:

$$
p=\rho R_{s} T,
$$

where $R_{s}$ is the specific ideal gas constant, and $T$ is the static, absolute temperature.

We will further assume the heat capacities are constant, so that the internal energy and enthalpy may be respectively written as

$$
\begin{gathered}
\varepsilon=c_{v} T, \\
h=c_{p} T,
\end{gathered}
$$

where $c_{v}$ is the volumetric heat capacity and $c_{p}$ is the isobaric heat capacity. Fluids which obey the ideal gas law and have constant heat capacities are called 
perfect gases [18].

\subsection{Nondimensionalization}

To further generalize our results we have presented much of our analyses and solutions in terms of non-dimensional quantities. We use the following scaled variables to non-dimensionalize the governing and auxillary equations:

$$
\hat{\boldsymbol{x}}^{*}=\frac{\hat{\boldsymbol{x}}}{x_{c}}, \hat{\boldsymbol{u}}^{*}=\frac{\hat{\boldsymbol{u}}}{u_{c}}, p^{*}=\frac{p}{\rho_{c} x_{c} u_{c} \Omega_{c}}, \boldsymbol{\Omega}^{*}=\frac{\boldsymbol{\Omega}}{\Omega_{c}}, T^{*}=\frac{T}{T_{c}}, \rho^{*}=\frac{\rho}{\rho_{c}}, \nabla^{*}=x_{c} \nabla
$$

Assuming the fluid is a perfect gas, the mass, momentum, and energy equations become

$$
\begin{gathered}
\nabla^{*} \cdot\left(\rho^{*} \hat{\boldsymbol{u}}^{*}\right)=0, \\
R o\left(\hat{\boldsymbol{u}}^{*} \cdot \nabla^{*}\right) \hat{\boldsymbol{u}}^{*}=-\frac{\nabla^{*} p^{*}}{\rho^{*}}+2 \hat{\boldsymbol{u}}^{*} \times \boldsymbol{\Omega}^{*}-\frac{1}{R o}\left(\hat{\boldsymbol{x}}^{*} \times \mathbf{\Omega}^{*}\right) \times \mathbf{\Omega}^{*}, \\
\rho^{*} \operatorname{Ro} \hat{\boldsymbol{u}}^{*} \cdot \nabla^{*} T^{*}=-\gamma(\gamma-1) M a^{2} p^{*}\left(\nabla^{*} \cdot \hat{\boldsymbol{u}}^{*}\right),
\end{gathered}
$$

where the relevant dimensionless groups are defined in Table 1 .

Using the same scaled variables the ideal gas Equation (4) becomes

$$
p^{*}=\frac{R o}{\gamma M a^{2}} \rho^{*} T^{*} .
$$

\section{Rotating Duct}

This section derives the general solution for compressible flow inside a rotating duct under the following assumptions:

1) constant thermophysical properties,

2) steady rotation about the $z$-axis: $\Omega=\omega \hat{\boldsymbol{k}}$,

3) steady flow,

4) subsonic flow,

5) unidirectional flow along the $\hat{x}$-axis such that $\hat{\boldsymbol{u}}=\hat{u} \hat{\boldsymbol{i}}$,

6) inviscid,

7) adiabatic, and

8) negligible heat conduction.

Based on these assumptions we have neglected any influences listed by Lyman [14] which may change the rothalpy inside the duct. A schematic of the duct under consideration is shown in Figure 1.

\subsection{Constant Cross-Section}

If the cross-sectional area of the duct is constant, the steady, non-dimensional conservation equations of mass, momentum, and energy reduce to

$$
\begin{gathered}
\hat{u}^{*} \frac{\mathrm{d} \rho^{*}}{\mathrm{~d} \hat{x}^{*}}+\rho^{*} \frac{\mathrm{d} \hat{u}^{*}}{\mathrm{~d} \hat{x}^{*}}=0, \\
R o \hat{u}^{*} \frac{\mathrm{d} \hat{u}^{*}}{\mathrm{~d} \hat{x}^{*}}=-\frac{1}{\rho^{*}} \frac{\mathrm{d} p^{*}}{\mathrm{~d} \hat{x}^{*}}+\frac{\hat{x}^{*}}{R o},
\end{gathered}
$$




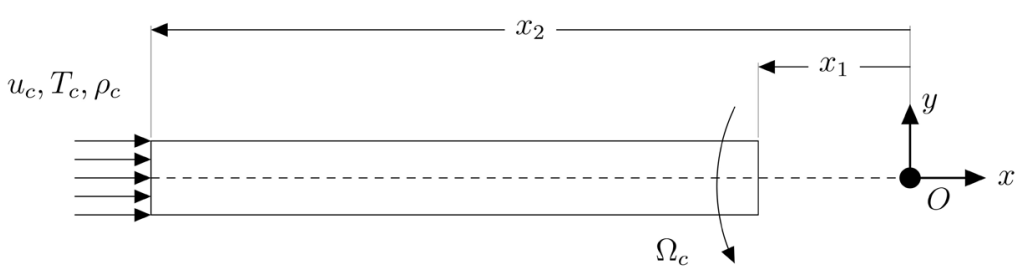

Figure 1. Schematic of the constant cross-section duct, rotating with a constant angular velocity about the origin $O$. Here the flow is shown moving from the outer position 2 to the inner position 1, however our analysis is independent of the flow direction. Furthermore, while we have chosen characteristic quantities at position 2 , the choice is arbitrary, as long as they are all at the same location.

Table 1. Relevant dimensionless groups.

$$
\begin{array}{rlrl}
R o & =\frac{u_{c}}{\Omega_{c} x_{c}} & & \text { Rossby number } \\
M a^{2} & =\frac{u_{c}^{2}}{\gamma R_{s} T_{c}} & \text { Mach number } \\
\gamma & =\frac{c_{p}}{c_{v}} & \text { Heat capacity ratio }
\end{array}
$$

$$
\operatorname{Ro} \rho^{*} \hat{u}^{*} \frac{\mathrm{d} T^{*}}{\mathrm{~d} \hat{x}^{*}}=-\gamma(\gamma-1) M a^{2} p^{*} \frac{\mathrm{d} \hat{u}^{*}}{\mathrm{~d} \hat{x}^{*}} .
$$

Use of the ideal gas law allows Equations (11) and (13) to be simplified and solved through direct integration.

$$
\begin{gathered}
\rho^{*}=\frac{C}{\hat{u}^{*}}, \\
T^{*}=\frac{D}{\hat{u}^{* \gamma-1}},
\end{gathered}
$$

where $C$ and $D$ are constants of integration. The pressure distribution is therefore given by

$$
p^{*}=\frac{R o}{\gamma M a^{2}} \frac{C D}{\hat{u}^{* \gamma}} .
$$

Solving Equation (12) requires substitution of 14 and 16 to obtain the differential equation

$$
\hat{u}^{*} \frac{\mathrm{d} \hat{u}^{*}}{\mathrm{~d} \hat{x}^{*}}=\frac{1}{M a^{2}} \frac{D}{\hat{u}^{* \gamma}} \frac{\mathrm{d} \hat{u}^{*}}{\mathrm{~d} \hat{x}^{*}}+\frac{\hat{x}^{*}}{R o^{2}},
$$

whose solution is

$$
\frac{\hat{u}^{* 2}}{2}+\frac{1}{(\gamma-1) M a^{2}} \frac{D}{\hat{u}^{* \gamma-1}}-\frac{\hat{x}^{* 2}}{2 R o^{2}}=E .
$$

Equation (18) is an expression of Bernoulli's theorem in a rotating framework. Inserting the boundary conditions $T^{*}(-1)=1$, and $\hat{u}^{*}(-1)=1$ yields 


$$
\begin{gathered}
T^{*}=\frac{1}{\hat{u}^{*} \gamma-1}, \\
\frac{1}{2}\left(\hat{u}^{* 2}-1\right)+\frac{1}{(\gamma-1) M a^{2}}\left(\frac{1}{\hat{u}^{*} \gamma-1}-1\right)-\frac{1}{2 R o^{2}}\left(\hat{x}^{* 2}-1\right)=0 .
\end{gathered}
$$

It is interesting to note that the velocity and temperature profiles are completely independent of the pressure and density. Only the inlet temperature and velocity boundary conditions influence the solution. Equation (20) may also be re-dimensionalized for better understanding of each of the terms:

$$
\underbrace{\frac{\hat{u}^{2}-\hat{u}_{c}^{2}}{2}}_{\begin{array}{c}
\text { linear } \\
\text { kineticenergy }
\end{array}}+\underbrace{c_{p}\left(T-T_{c}\right)}_{\text {enthalpy }}-\underbrace{\frac{\omega^{2}\left(\hat{x}^{2}-x_{c}^{2}\right)}{2}}_{\begin{array}{c}
\text { rotational } \\
\text { kineticenergy }
\end{array}}=0 .
$$

When the Mach and Rossby numbers are very small, the linear kinetic energy term in Equation (20) may be neglected and the temperature profile reduces to

$$
T^{*}=1-\frac{(\gamma-1) M a^{2}}{2 R o^{2}}\left(1-\hat{x}^{* 2}\right) .
$$

Re-dimensionalizing Equation (22) and evaluating at $\hat{x}=0$ yields the temperature distribution found by Polihronov and Straatman [1]:

$$
T_{c}-T=\frac{\omega^{2} x_{c}^{2}}{2 c_{p}} .
$$

This indicates their analysis has implicitly assumed the compressibility of the fluid is small, and the rotational energy of the fluid is large.

We have performed several computational fluid dynamics (CFD) simulations of rotating duct model using ANSYS-CFX ( $)$ software [19] to demonstrate the accuracy of Equations (15) and (18) over Equation (22). A 1D mesh was generated for a straight square duct containing $10^{3}$ evenly spaced grid points. Air was chosen as the working fluid, with a heat capacity ratio $\gamma=1.4$, and a free slip boundary condition was enforced at each of the duct walls. The average residuals for the solution were converged within $10^{-4}$. The results are shown in Figure 2. A maximum error of $0.03 \%$ was observed between Equation (18) and the CFD velocity profile, and a maximum error of $5 \times 10^{-4} \%$ was observed between Equation (15) and the CFD temperature profile.

\subsection{Arbitrary Cross-Sectional Area}

We will now generalize the above results to a duct of varying cross section $A(\hat{x})$. Analyzing a thin slice $\hat{x}$ of a straight duct aligned with the $\hat{x}$ axis where the free-slip boundary condition is applied at the duct walls leads to the following governing equations:

$$
\begin{gathered}
\frac{1}{\rho} \frac{\mathrm{d} \rho}{\mathrm{d} \hat{x}}+\frac{1}{A} \frac{\mathrm{d} A}{\mathrm{~d} \hat{x}}+\frac{1}{\hat{u}} \frac{\mathrm{d} \hat{u}}{\mathrm{~d} \hat{x}}=0, \\
\hat{u} \frac{\mathrm{d} \hat{u}}{\mathrm{~d} \hat{x}}=-\frac{1}{\rho} \frac{\mathrm{d} p}{\mathrm{~d} \hat{x}}+x \omega^{2},
\end{gathered}
$$



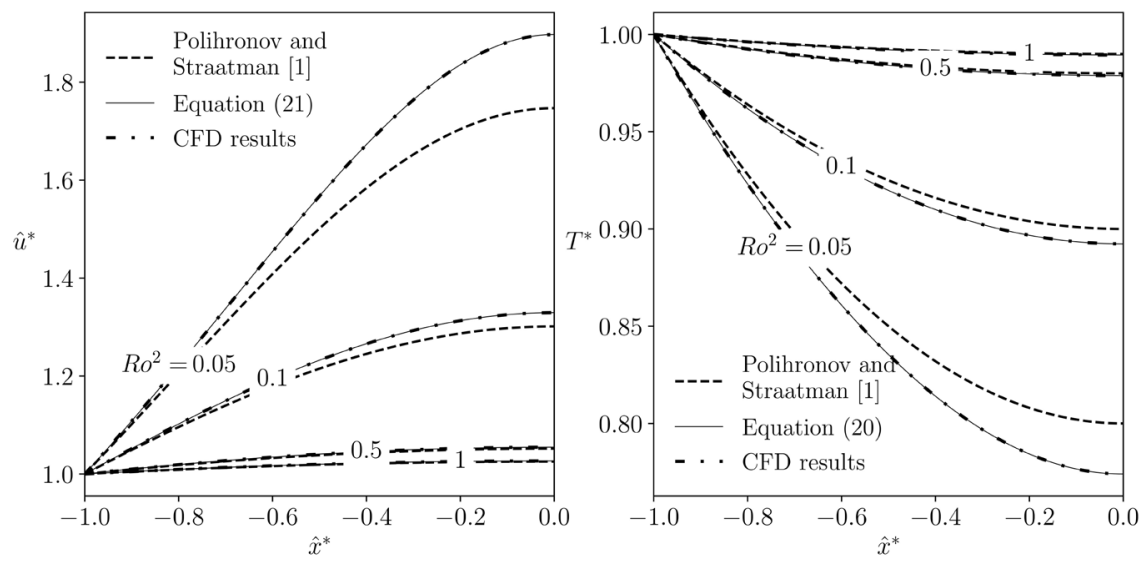

Figure 2. Plots of non-dimensional velocity and temperature in a straight duct with $M a^{2}=0.05$ and $\gamma=1.4$.

$$
c_{v} \frac{\mathrm{d} T}{\mathrm{~d} \hat{x}}=-\frac{p}{\rho}\left(\frac{1}{A} \frac{\mathrm{d} A}{\mathrm{~d} \hat{x}}+\frac{1}{\hat{u}} \frac{\mathrm{d} \hat{u}}{\mathrm{~d} \hat{x}}\right) .
$$

Invoking the ideal gas Equation (10), introducing the scaled cross-sectional area $A^{*}\left(\hat{x}^{*}\right)=A(\hat{x}) / A_{c}$, and non-dimensionalizing $24-26$ yields:

$$
\begin{gathered}
\frac{1}{\rho^{*}} \frac{\mathrm{d} \rho^{*}}{\mathrm{~d} \hat{x}^{*}}+\frac{1}{A^{*}} \frac{\mathrm{d} A^{*}}{\mathrm{~d} \hat{x}^{*}}+\frac{1}{\hat{u}^{*}} \frac{\mathrm{d} \hat{u}^{*}}{\mathrm{~d} \hat{x}^{*}}=0, \\
R o \hat{u}^{*} \frac{\mathrm{d} \hat{u}^{*}}{\mathrm{~d} \hat{x}^{*}}=-\frac{1}{\rho^{*}} \frac{\mathrm{d} p^{*}}{\mathrm{~d} \hat{x}^{*}}+\frac{\hat{x}^{*}}{R o}, \\
\frac{1}{T^{*}} \frac{\mathrm{d} T^{*}}{\mathrm{~d} \hat{x}^{*}}=-(\gamma-1)\left(\frac{1}{A^{*}} \frac{\mathrm{d} A^{*}}{\mathrm{~d} \hat{x}^{*}}+\frac{1}{\hat{u}^{*}} \frac{\mathrm{d} \hat{u}^{*}}{\mathrm{~d} \hat{x}^{*}}\right) .
\end{gathered}
$$

Equations (27) and (29) may be solved by direct integration, and Equation (10) may be used to obtain an expression for the pressure distribution:

$$
\begin{gathered}
\rho^{*}=\frac{C}{A^{*} \hat{u}^{*}}, \\
T^{*}=\frac{D}{\left(A^{*} \hat{u}^{*}\right)^{\gamma-1}}, \\
p^{*}=\frac{R o}{\gamma M a^{2}} \frac{C D}{\left(A^{*} \hat{u}^{*}\right)^{\gamma}} .
\end{gathered}
$$

Solving Equation (28) requires the use of Equations (30) and (32) to obtain

$$
\left(\hat{u}^{*}-\frac{D}{M a^{2}} \frac{1}{\left(A^{*} \hat{u}^{*}\right)^{\gamma-1} \hat{u}^{*}}\right) \frac{\mathrm{d} \hat{u}^{*}}{\mathrm{~d} \hat{x}^{*}}-\frac{D}{M a^{2}} \frac{1}{\left(A^{*} \hat{u}^{*}\right)^{\gamma-1} A^{*}} \frac{\mathrm{d} A^{*}}{\mathrm{~d} \hat{x}^{*}}-\frac{\hat{x}^{*}}{R o^{2}}=0 .
$$

Equation (33) may be solved using the method of exact differentials, yielding

$$
\frac{\hat{u}^{* 2}}{2}+\frac{1}{M a^{2}(\gamma-1)} \frac{D}{\left(\hat{u}^{*} A^{*}\right)^{\gamma-1}}-\frac{\hat{x}^{* 2}}{2 R o^{2}}=E .
$$


Three boundary conditions are required to evaluate constants $C, D$ and $E$. If the duct area is constant (i.e. $A(\hat{x})=A_{c}$ ) then $A^{*}=1$ and Equations (30), (31), (32) and (34) reduce to the solutions for a constant cross-section duct; Equations (14), (15), (16) and (18) respectively. In addition, we note that Equation (34) is in complete agreement with Equation (9) in Ref. [12].

To confirm this result, we have conducted several CFD simulations and compared the computed profiles to Equations (31) and (34). These simulations were similar to those described in section 1 unless otherwise noted. The geometry under consideration is the straight square duct depicted in Figure 3 whose cross-sectional area is given by

$$
A^{*}\left(\hat{x}^{*}\right)=\left(3\left|\hat{x}^{*}\right|+4\right)^{2} .
$$

A $1 \mathrm{D}$ mesh of constant grid spacing with $10^{3}$ grid points was generated. The solution was again computed using ANSYS CFX () [19]. Solutions were converged when the average residuals were reduced below $10^{-4}$. The results have been plotted in Figure 4. A maximum error of $0.6 \%$ was observed between Equation (34) and the CFD results while a maximum error of $0.004 \%$ was observed between Equation (31) and the CFD results.

\section{Rotating Passage}

In this section we will further generalize the above results to an arbitrarily curved passage defined by the parameterization

$$
\boldsymbol{p}(a)=X(a) \hat{\boldsymbol{i}}+Y(a) \hat{\boldsymbol{j}}+Z(a) \hat{\boldsymbol{k}} .
$$

and the scaled path vector is given by $\boldsymbol{p}^{*}=\boldsymbol{p} / x_{c}$. The components of $\boldsymbol{p}$ may be any well-behaved functions, producing, for example, the path shown in Figure 5 .

The following derivation requires that the axis of rotation contains the origin of the co-ordinate system on which $p^{*}$ is defined. The unit tangent vector parallel to the path $\boldsymbol{p}$ is given by

$$
\boldsymbol{t}=\frac{\boldsymbol{p}^{\prime}(a)}{\left\|\boldsymbol{p}^{\prime}(a)\right\|}=\frac{\boldsymbol{p}^{* \prime}(a)}{\left\|\boldsymbol{p}^{* \prime}(a)\right\|} .
$$

Similarly to the previous derivations, we will neglect the velocity variation across the duct, and assume the velocity at each point is parallel to the unit tangent vector:

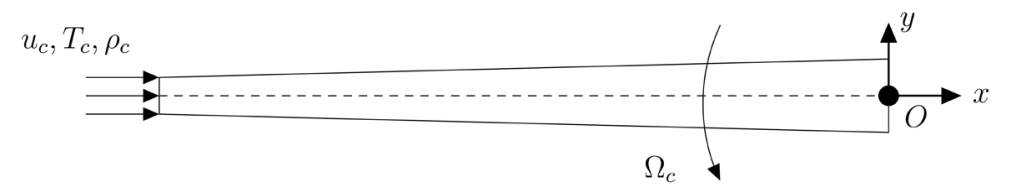

Figure 3. Schematic of the varying cross-section duct, rotating with a constant angular velocity $\Omega_{c}$ about the origin $O$. The area as a function of the $\hat{x}$ co-ordinate is given by Equation (35). 

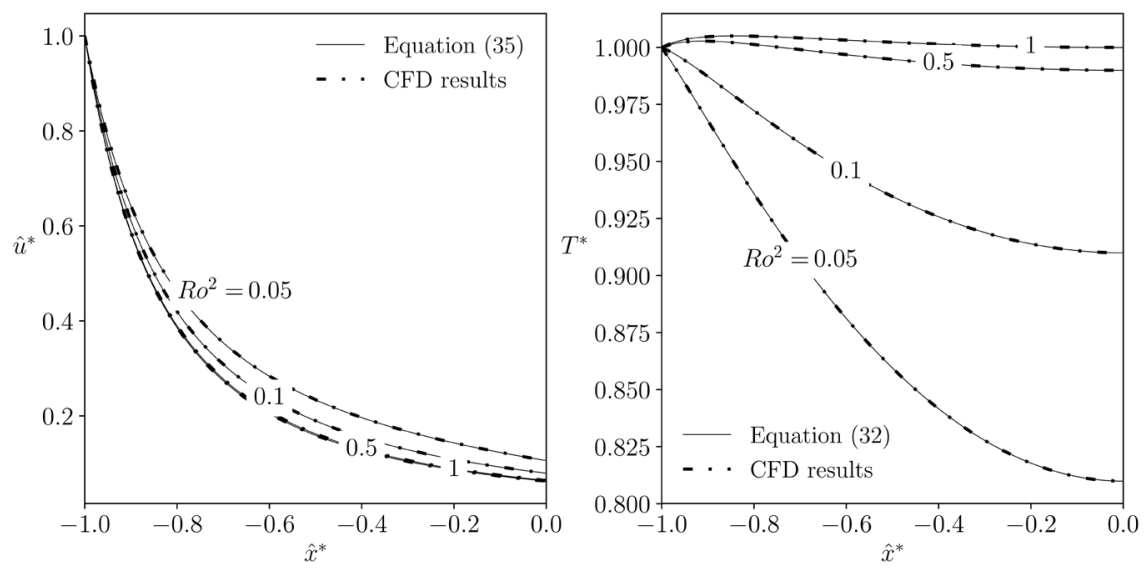

Figure 4. Plots of non-dimensional velocity and temperature of a duct with cross-sectional area varying in accordance with 36 with $M a^{2}=0.05$ and $\gamma=1.4$.

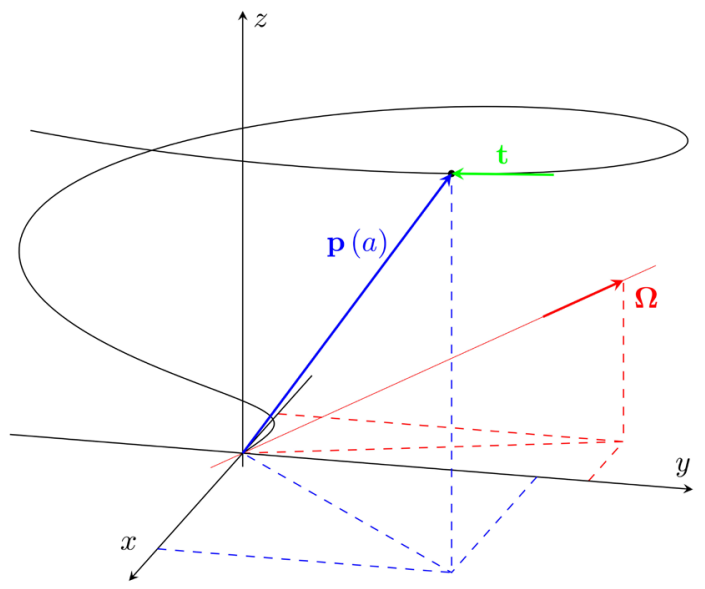

Figure 5 . An arbitrary path defined by $p(a)$, rotating about $\Omega$.

$$
\frac{\hat{\boldsymbol{u}}^{*}}{\bar{u}^{*}}=\boldsymbol{t}
$$

where $\bar{u}^{*}=\left\|\hat{\boldsymbol{u}}^{*}\right\|$.

\subsection{Constant Cross-Section}

The following steps apply when the duct cross-sectional area is constant along the path. If we have some quantity $\phi\left(p^{*}(a)\right)$, its total derivative is

$$
\frac{\mathrm{d} \phi}{\mathrm{d} a}=\frac{\partial \phi}{\partial x} \frac{\mathrm{d} x}{\mathrm{~d} a}+\frac{\partial \phi}{\partial y} \frac{\mathrm{d} y}{\mathrm{~d} a}+\frac{\partial \phi}{\partial z} \frac{\mathrm{d} z}{\mathrm{~d} a}=\nabla \phi \cdot \boldsymbol{p}^{* \prime}(a)=\left(\boldsymbol{p}^{* \prime}(a) \cdot \nabla\right) \phi .
$$

Furthermore, given that all quantities are defined only on the path $p(a)$, any gradient (e.g. $\nabla \phi)$ is parallel to the unit tangent vector:

$$
\nabla \phi=c \frac{\mathrm{d} \phi}{\mathrm{d} a} \boldsymbol{t},
$$

for some unknown value $c$. We will choose $c=1 /\left\|p^{* \prime}(a)\right\|$ to satisfy Equation (39). Substituting Equations (38) and (40) into the governing Equations (7) and 
(9) yields (after some manipulation)

$$
\begin{gathered}
\frac{1}{\rho^{*}} \frac{\mathrm{d} \rho^{*}}{\mathrm{~d} a}+\frac{1}{\bar{u}^{*}} \frac{\mathrm{d} \bar{u}^{*}}{\mathrm{~d} a}=0, \\
\frac{1}{T^{*}} \frac{\mathrm{d} T^{*}}{\mathrm{~d} a}+\frac{(\gamma-1)}{\bar{u}^{*}} \frac{\mathrm{d} \bar{u}^{*}}{\mathrm{~d} a}=0 . \\
\left(\bar{u}^{*}+\frac{D}{\gamma M a^{2} \bar{u}^{* \gamma}}\right) \frac{R o}{\left\|\boldsymbol{p}^{* \prime}\right\|} \frac{\mathrm{d} \bar{u}^{*}}{\mathrm{~d} a} \boldsymbol{t}+\frac{R o}{\left\|\boldsymbol{p}^{* \prime}\right\|} \bar{u}^{* 2} \boldsymbol{t}^{\prime} \\
=2 \bar{u}^{*}\left(\boldsymbol{t} \times \boldsymbol{\Omega}^{*}\right)+\frac{1}{R o}\left[\boldsymbol{p}^{*}-\left(\boldsymbol{p}^{*} \cdot \boldsymbol{\Omega}^{*}\right) \boldsymbol{\Omega}^{*}\right] .
\end{gathered}
$$

Solving 41 and 42 yields Equations (14) and (15), respectively. The ideal gas law may be expressed using Equation (16).

To obtain a general solution for the velocity profile we will take the dot product of Equation (43) with $\boldsymbol{t}$. Since $\boldsymbol{t}$ and $\boldsymbol{t}^{\prime}$ are orthogonal, the second term on the left hand side must vanish. Furthermore, the first term on the right hand side also evaluates to zero, since it contains a triple scalar product with two parallel vectors. The remaining equation is given by

$$
\left(\bar{u}^{*}+\frac{D}{\gamma M a^{2} \bar{u}^{* \gamma}}\right) \bar{u}^{*} a=\frac{g^{\prime}(a)}{R o^{2}},
$$

where

$$
g^{\prime}(a)=\boldsymbol{p}^{*} \cdot \boldsymbol{p}^{* \prime}-\left(\boldsymbol{p}^{*} \cdot \boldsymbol{\Omega}^{*}\right)\left(\boldsymbol{p}^{* \prime} \cdot \boldsymbol{\Omega}^{*}\right) .
$$

Equation (44) may be solved using direct integration:

$$
\frac{\bar{u}^{* 2}}{2}+\frac{1}{(\gamma-1) M a^{2}} \frac{D}{\bar{u}^{* \gamma-1}}-\frac{g(a)}{R o^{2}}=E .
$$

\subsection{Arbitrary Cross-Sectional Area}

For a rotating passage of arbitrarily varying cross-sectional area $A(a)$, and $A^{*}(a)=A(a) / A_{c}$ we must include $A^{*}(a)$ in a manner similar to Section 2. The density, temperature, and pressure profiles are given by Equations (30), (31), and (32), respectively. The velocity profile is given by

$$
\frac{\bar{u}^{* 2}}{2}+\frac{1}{(\gamma-1) M a^{2}} \frac{D}{\left(\bar{u}^{*} A^{*}\right)^{\gamma-1}}-\frac{g(a)}{R o^{2}}=E .
$$

\subsection{Radius as the Parameter}

In light of this result, we are interested to see if we can make any statements about the function $g(a)$. Consider the following arbitrary curve in a cylindrical co-ordinate system whose $z$-axis is coincident with the axis of rotation:

$$
\begin{gathered}
\theta=\theta(r), \\
z=\zeta(r, \theta(r)) .
\end{gathered}
$$

We will proceed with the parameterization $r=a$ : 


$$
\boldsymbol{p}^{*}=a[\cos (\theta(a)) \hat{\boldsymbol{i}}+\sin (\theta(a)) \hat{\boldsymbol{j}}]+\zeta(a, \theta(a)) \hat{\boldsymbol{k}} .
$$

Invoking definition 46 reveals $g^{\prime}(a)=a$ whenever $\theta^{\prime}(a)$ is well-behaved over the desired range of a. Under these circumstances, Equation (47) collapses to 34, and we conclude that the flow speed $\bar{u}^{*}$ at any point in a constant cross-section rotating passage under isentropic conditions is a function of the radial position only.

\section{Work}

One parameter of particular interest is the work derived from a radial turbine (or the work required to drive a radial compressor). In a straight duct, the work is most easily found by writing an energy balance over a control volume enveloping a section of the passage between two points:

$$
\dot{W}-\dot{Q}=\dot{E}_{\mathrm{st}}+\dot{m}_{2}\left(h_{2}+\frac{u_{2}^{2}}{2}\right)-\dot{m}_{1}\left(h_{1}+\frac{u_{1}^{2}}{2}\right) .
$$

Because the flow is adiabatic and steady we may neglect the heat transfer $\dot{Q}$ and transient energy storage $\dot{E}_{\text {st }}$ respectively. Furthermore we recognize that $\dot{m}_{1}=\dot{m}_{2}=\dot{m}$ and insert Equation (6). With these simplifications, we have

$$
\dot{W}=\dot{m}\left(c_{p}\left(T_{2}-T_{1}\right)+\frac{1}{2}\left(u_{2}^{2}-u_{1}^{2}\right)\right) .
$$

In a straight, radial duct such as the one shown in Figure 1 or the duct shown in Figure 3 we recognize that the velocity in the stationary frame is the vector sum of the in-frame velocity and the local tangential velocity of the duct $u(\hat{x})=\sqrt{\hat{u}^{2}+\omega^{2} \hat{x}^{2}}$ :

$$
\begin{aligned}
& \dot{W}=\dot{m}\left(c_{p} T_{2}+\frac{\left(\sqrt{\hat{u}_{2}^{2}+\omega^{2} \hat{x}_{2}^{2}}\right)^{2}}{2}-c_{p} T_{1}-\frac{\left(\sqrt{\hat{u}_{1}^{2}+\omega^{2} \hat{x}_{1}^{2}}\right)^{2}}{2}\right) \\
&=\dot{m}\left(c_{p}\left(T_{2}-T_{1}\right)+\frac{\hat{u}_{2}^{2}-\hat{u}_{1}^{2}}{2}+\frac{\omega^{2}\left(\hat{x}_{2}^{2}-\hat{x}_{1}^{2}\right)}{2}\right) \\
&=\dot{m} \omega^{2}\left(\hat{x}_{2}^{2}-\hat{x}_{1}^{2}\right) \\
& \dot{W}^{*}=\frac{\left(\hat{x}_{2}^{* 2}-\hat{x}_{1}^{* 2}\right)}{R o^{2}} .
\end{aligned}
$$

where $\dot{W}^{*}=\dot{W} / \dot{m} \hat{u}_{c}^{2}$ and Equation (21) has been used. Equation (53) might be rewritten in terms of a duct tip speed $c=\omega \hat{x}_{2}$, so that where $\hat{x}_{1}=0$, the work transferred to/from the passage is given by

$$
\dot{W}=\dot{m} c^{2} .
$$

Equation (55) is the rate form of the angular rocket propulsion equation developed by Polihronov and Straatman [2].

In a curved passage we must express the velocity in the stationary frame as $u(a)=\|\bar{u} \boldsymbol{t} \boldsymbol{\Omega} \times \boldsymbol{p}\|$. Substituting this expression into Equation (52) gives 


$$
\dot{W}=\dot{m}\left(c_{p}\left(T_{2}-T_{1}\right)+\frac{1}{2}\left(\left\|\bar{u}_{2} \boldsymbol{t}_{2}+\boldsymbol{\Omega} \times \boldsymbol{p}_{2}\right\|^{2}-\left\|\bar{u}_{1} \boldsymbol{t}_{1}+\boldsymbol{\Omega} \times \boldsymbol{p}_{1}\right\|^{2}\right)\right) .
$$

This equation cannot be reduced any further without knowing the form of $p^{*}$.

\section{Choked Flow Limitations}

Several assumptions have been employed to arrive at the density, temperature, pressure, and velocity profiles of the above sections. These profiles are therefore only valid for particular combinations of Rossby and Mach numbers. While each of the assumptions listed at the beginning of Section 2 merit their own discussion, in this work we will restrict our analysis to the sonic limit. If the flow transitions from subsonic to supersonic at any point in a rotating passage, there will inevitably be a shock at some point downstream as it again becomes subsonic. Shocks are highly irreversible and therefore undesirable in many applications, therefore it is of great interest to prevent the flow from transitioning in the first place. The next two subsections identify the conditions under which the flow transitions in rotating passages, and develop the appropriate constraints on the selection of $R o$ and $M a$.

\subsection{Sonic Limitation in the Shroud}

Previously the adiabatic duct has been experimentally validated through injecting air tangentially into a circular passage surrounding a rotating disk and allowing the air to expand through radial passages in the disk [20]. In this configuration the Mach number of the flow through the shroud, $M a_{\mathrm{S}}$, should be less than 1:

$$
\begin{gathered}
M a_{\mathrm{S}}<1 \\
\frac{u_{2}}{\sqrt{\gamma R_{s} T_{2}}}<1 \\
\frac{\omega^{2} \hat{x}_{2}}{\sqrt{\gamma R_{s} T_{2}}}<1 \\
\frac{M a^{2}}{R o^{2}}<1 \\
M a^{2}<R o^{2} .
\end{gathered}
$$

\subsection{Stagnation Properties}

In addition, we must ensure the flow does not transition within the passage itself, a state characterized by the presence of choked flow within the passage. To properly define this constraint we must first define several quantities before the topic can be addressed.

First, recall the total enthalpy in the stationary frame is defined as the total energy of a flowing stream per unit mass [21]: 


$$
h_{0}=h+\frac{u^{2}}{2} \text {. }
$$

If the fluid is assumed to be a perfect gas, the total temperature is found by invoking Equation (6)

$$
T_{0}=T+\frac{u^{2}}{2 c_{p}}=T+\frac{\hat{u}^{2}+\omega^{2} \hat{r}^{2}}{2 c_{p}} .
$$

Equation 60 is similar to Equation (17-4) in the thermodynamics text by Cengel and Boles [3], with the inclusion of the rotational energy per unit mass. This quantity is useful in stationary flows because it is constant over isentropic processes.

In contrast, the total temperature in the rotating frame may be defined as:

$$
\hat{T}_{0}=T+\frac{\hat{u}^{2}}{2 c_{p}}
$$

To see if either of these parameters are constant in the rotating duct problem we insert the temperature profile (Equation (31)) into the velocity profile (Equation (47)) and re-dimensionalize:

$$
\frac{\hat{u}^{2}}{2 c_{p}}+T-\frac{\omega^{2} \hat{r}^{2}}{2 c_{p}}=\bar{T}_{0} .
$$

Rearranging the above equation gives

$$
\bar{T}_{0}=T+\frac{\hat{u}^{2}-\omega^{2} \hat{r}^{2}}{2 c_{p}} .
$$

We have called $\bar{T}_{0}$ the stagnation temperature, as this is the temperature which is attained if the fluid is brought to rest isentropically (while exchanging some energy with the walls of the passage). We have also replaced the parameter $a$ with the co-ordinate $\hat{r}$, to emphasize that this quantity is the radial distance from the axis of rotation. Upon comparing Equations (59)-(61) it's clear that neither the total temperature in the stationary frame nor the total temperature in the relative frame are constant along the passage, while the stagnation temperature, $\bar{T}_{0}$, is. Readers familiar with turbomachinery analysis will recognize the quantity $\bar{T}_{0} c_{p}$ as the rothalpy [7]. Notice that the total temperature and stagnation temperature are not equal in general; i.e. $\hat{T}_{0} \neq \bar{T}_{0}$.

The isentropic gas equations may be used to find relationships between stagnation and static pressure and density:

$$
\begin{aligned}
& \frac{\bar{p}_{0}}{p}=\left(\frac{\bar{T}_{0}}{T}\right)^{\gamma /(\gamma-1)}, \\
& \frac{\bar{\rho}_{0}}{\rho}=\left(\frac{\bar{T}_{0}}{T}\right)^{1 /(\gamma-1)} .
\end{aligned}
$$

Furthermore, we can use Equation (61) to define the ratio of stagnation to static temperature in terms of dimensionless numbers: 


$$
\begin{gathered}
\frac{\bar{T}_{0}}{T}=1+\frac{\hat{u}^{2}-\omega^{2} \hat{r}^{2}}{2 c_{p} T}, \\
\frac{\bar{T}_{0}}{T}=\frac{1+\frac{\gamma-1}{2} M a_{L}^{2}}{1+\frac{\gamma-1}{2}\left(\frac{\hat{r}}{\hat{r}_{c}}\right)^{2} M a_{t}^{2}},
\end{gathered}
$$

where $M a_{L}$ is a local Mach number and $M a_{t}=\omega x_{c} / \sqrt{\gamma R_{s} \bar{T}_{0}}$ is the tip Mach number. Equation 64 reduces to its stationary counterpart (Equations (16)-(17) in Ref. [21]) when $\omega=0$.

In addition, we evaluate Equation (47) at the location $\hat{r}^{*}=1$ to devise two useful relationships between $M a, R o, \gamma$, and $M a_{t}$ :

$$
\begin{gathered}
\frac{1}{2}+\frac{1}{(\gamma-1) M a^{2}}-\frac{1}{2 R o^{2}}=\frac{1}{(\gamma-1) M a_{t}^{2} R o^{2}}, \\
\frac{M a^{2}}{R o^{2} M a_{t}^{2}}=\frac{\bar{T}_{0}}{T_{c}}=\frac{1+\frac{\gamma-1}{2} M a^{2}}{1+\frac{\gamma-1}{2} M a_{t}^{2}} .
\end{gathered}
$$

\subsection{Choked Flow}

Using the above definitions, the choked flow condition may be identified. The mass flow rate at any location in a radial passage is given by

$$
\dot{m}=\rho A \hat{u}=p A \sqrt{\frac{\gamma}{R T}} M a_{L} .
$$

Using property ratios 65 and 63 and simplifying yields

$$
\dot{m}=\bar{p}_{0} A \sqrt{\frac{\gamma}{R \bar{T}_{0}}} M a_{L}\left[\frac{1+\frac{\gamma-1}{2}\left(\frac{\hat{r}}{\hat{r}_{c}}\right)^{2} M a_{t}^{2}}{1+\frac{\gamma-1}{2} M a_{L}^{2}}\right]^{(\gamma+1) /[2(\gamma-1)]} .
$$

We can also define the maximum possible mass flow rate for any given duct, by differentiating Equation (68) with respect to $M a_{L}$ and setting the result equal to 0 , which yields $M a_{L}=1$. Inserting this restriction into Equation (68) yields the critical, or choked mass flow:

$$
\dot{m}_{\mathrm{Ch}}=\bar{p}_{0} A \sqrt{\frac{\gamma}{R \bar{T}_{0}}}\left[\frac{2}{\gamma+1}\left(1+\frac{\gamma-1}{2}\left(\frac{\hat{r}}{\hat{r}_{c}}\right)^{2} M a_{t}^{2}\right)\right]^{(\gamma+1) /[2(\gamma-1)]} .
$$

We can nondimensionalize with $\dot{m}_{\mathrm{Ch}}=\dot{m}_{\mathrm{Ch}}^{*} \rho_{c} A_{c} \hat{u}_{c}$ :

$$
\dot{m}_{\mathrm{Ch}}^{*}=\frac{\bar{\rho}_{0}}{\rho_{c}} \sqrt{\frac{\bar{T}_{0}}{T_{c}}} \frac{A^{*}}{M a}\left[\frac{2}{\gamma+1}\left(1+\frac{\gamma-1}{2} \hat{r}^{* 2} M a_{t}^{2}\right)\right]^{(\gamma+1) /[2(\gamma-1)]} .
$$

Using Equations (63) and (64), the property ratios in the above equation may be cast in terms of the global Mach number and tip Mach number: 


$$
\dot{m}_{\mathrm{Ch}}^{*}=\frac{A^{*}}{M a}\left[\frac{2}{\gamma+1} \frac{1+\frac{\gamma-1}{2} M a^{2}}{1+\frac{\gamma-1}{2} M a_{t}^{2}}\left(1+\frac{\gamma-1}{2} \hat{r}^{* 2} M a_{t}^{2}\right)\right]^{(\gamma+1) /[2(\gamma-1)]} .
$$

Equation (71) represents the maximum possible mass flow rate at any radial location. Notice $\dot{m}_{\mathrm{Ch}}^{*}$ varies with the radial co-ordinate $\hat{r}^{*}$. If, at any location, $\dot{m}_{\mathrm{Ch}}^{*}>1$, the flow will be choked in the passage.

While expression 71 is useful, we desire a simpler test to determine whether the flow is choked. Regardless of the area profile the critical mass flow rate in the passage is dictated by the location of minimum choked flow, that is, where Equation (71) is minimized. We begin by differentiating with respect to $\hat{r}^{*}$ :

$$
\begin{aligned}
\frac{\mathrm{d} \dot{m}_{\mathrm{Ch}}^{*}}{\mathrm{~d} \hat{r}^{*}}= & A^{*} B \frac{\gamma+1}{2}\left[1+\frac{\gamma-1}{2} \hat{r}^{* 2} M a_{t}^{2}\right]^{(\gamma+1) /[2(\gamma-1)]-1} \hat{r}^{*} M a_{t}^{2} \\
& +\frac{\mathrm{d} A^{*}}{\mathrm{~d} \hat{r}^{*}} B\left[1+\frac{\gamma-1}{2} \hat{r}^{* 2} M a_{t}^{2}\right]^{(\gamma+1) /[2(\gamma-1)]} .
\end{aligned}
$$

where we have defined the parameter $B$ for compactness:

$$
B=\frac{1}{M a}\left[\frac{2}{\gamma+1} \frac{1+\frac{\gamma-1}{2} M a^{2}}{1+\frac{\gamma-1}{2} M a_{t}^{2}}\right]^{(\gamma+1) /[2(\gamma-1)]} .
$$

Setting Equation (72) equal to 0 yields

$$
\frac{1}{A^{*}} \frac{\mathrm{d} A^{*}}{\mathrm{~d} \hat{r}^{*}}=-\frac{\gamma+1}{2} \frac{\hat{r}^{*} M a_{t}^{2}}{1+\frac{\gamma-1}{2} \hat{r}^{* 2} M a_{t}^{2}} .
$$

\subsubsection{Constant Cross-Section Duct}

For the case when $A^{*}=1$, the above equation suggests the only extrema is at $\hat{r}^{*}=0$. By differentiating 73 again and inserting $\hat{r}=0$ gives

$$
\frac{\mathrm{d}^{2} \dot{m}_{\mathrm{Ch}}^{*}}{\mathrm{~d} \hat{r}^{* 2}}=A^{*} B \frac{\gamma+1}{2} M a_{t}^{2} .
$$

Since each of the terms in the above equation are positive, the concavity of 71 is positive at $\hat{r}^{*}=0$, confirming that $\hat{r}^{*}=0$ is a minima. Since it is the only extrema, it must be the global minimum, and therefore the location which determines the minimum choked mass flow rate for the duct of constant cross-section. Inserting $\hat{r}^{*}=0$ into Equation (71) yields

$$
\dot{m}_{\mathrm{Ch}, \min }^{*}=\frac{1}{M a}\left[\frac{2}{\gamma+1} \frac{1+\frac{\gamma-1}{2} M a^{2}}{1+\frac{\gamma-1}{2} M a_{t}^{2}}\right]^{(\gamma+1) /[2(\gamma-1)]} .
$$

Therefore, in order to ensure the flow is not choked, we require

$$
\dot{m}^{*}<\dot{m}_{\mathrm{Ch}, \min }^{*},
$$




$$
\begin{gathered}
1<\frac{1}{M a}\left[\frac{2}{\gamma+1} \frac{1+\frac{\gamma-1}{2} M a^{2}}{1+\frac{\gamma-1}{2} M a_{t}^{2}}\right]^{(\gamma+1) /[2(\gamma-1)]}, \\
1<\frac{1}{M a}\left[\frac{2}{\gamma+1} \frac{M a^{2}}{R o^{2} M a_{t}^{2}}\right]^{(\gamma+1) /[2(\gamma-1)]}, \\
\frac{2}{\gamma+1} M a^{4 /(\gamma+1)}>R o^{2} M a_{t}^{2} .
\end{gathered}
$$

Combining Equations (75) and (65) and re-arranging results in a cumbersome inequality in terms of $M a$ and $R o$, which has been plotted in Figure 6.

\subsubsection{Rotating Slice}

If the duct area varies with the equation $A^{*}=\hat{r}^{*}, \hat{r}^{*}>0 \quad$ (a rotating slice), Equation (73) reduces to $\hat{r}^{*}=i / \gamma M a_{t}$. Since there are no real solutions, there are no extrema on Equation (71), and the critical section for choked flow may be determined by comparing the choked mass flow rates at the inner and outer radii: $\hat{r}^{*}=\hat{r}_{1}^{*}, \hat{r}_{2}^{*}$ where $\hat{r}_{1}^{*}<\hat{r}_{2}^{*}$. Clearly, $\dot{m}_{\mathrm{Ch}}^{*}\left(\hat{r}_{1}^{*}\right)<\dot{m}_{\mathrm{Ch}}^{*}\left(\hat{r}_{2}^{*}\right)$, and the maximum mass flow rate is

$$
\dot{m}_{\mathrm{Ch}, \min }^{*}=\frac{\hat{r}_{1}^{*}}{M a}\left[\frac{2}{\gamma+1} \frac{1+\frac{\gamma-1}{2} M a^{2}}{1+\frac{\gamma-1}{2} M a_{t}^{2}}\left(1+\frac{\gamma-1}{2} \hat{r}_{1}^{* 2} M a_{t}^{2}\right)\right]^{(\gamma+1) /[2(\gamma-1)]} .
$$

\subsubsection{Critical Duct}

If Equation (71) is evaluated such that the flow is choked everywhere $\left(\dot{m}_{\mathrm{Ch}}^{*}=1\right)$, we can formulate the critical cross-sectional area profile:

$$
\begin{aligned}
A_{\mathrm{Ch}}^{*} & =M a\left[\frac{\gamma+1}{2} \frac{1}{1+\frac{\gamma-1}{2} M a^{2}} \frac{1+\frac{\gamma-1}{2} M a_{t}^{2}}{1+\frac{\gamma-1}{2} \hat{r}^{* 2} M a_{t}^{2}}\right]^{(\gamma+1) /[2(\gamma-1)]} \\
& =\frac{M a_{t}^{*}\left(M a_{t}, \hat{r}^{*}, \gamma\right)}{A_{t}^{*}(M a, \gamma)} .
\end{aligned}
$$

where we have recognized the appearance of the area ratio $A / A_{t}$, which has been defined for stationary ducts:

$$
A_{t}^{*}=\frac{A}{A_{t}}=\frac{1}{M a}\left[\frac{2}{\gamma+1}\left(1+\frac{\gamma-1}{2} M a^{2}\right)\right]^{(\gamma+1) /[2(\gamma-1)]} .
$$

We have also introduced a modified tip Mach number,

$$
M a_{t}^{*}=\left[\frac{1+\frac{\gamma-1}{2} M a_{t}^{2}}{1+\frac{\gamma-1}{2} \hat{r}^{* 2} M a_{t}^{2}}\right]^{(\gamma+1) /[2(\gamma-1)]} .
$$

Equation (78) has been tabulated for many values of $M a$ and $\gamma$ in many en- 
gineering texts such as Ref. [21]. The scale of the profile is determined by $A_{t}^{*}$, while the profile shape is determined by $M a_{t}^{*}$, which has been plotted in Figure 7. When $M a=1, A_{\mathrm{Ch}}^{*}=M a_{t}^{*}$ and the curves represent the minimum area required to avoid the choked flow condition. When $M a<1$, these curves are scaled by $1 / A_{t}^{*}$. Notice the required area decreases with increasing $\hat{r}^{*}$. We now propose an alternative method to determine whether the flow is choked in a known duct: plot the profiles $A^{*}\left(\hat{r}^{*}\right)$ and $A_{\mathrm{Ch}}^{*}\left(\hat{r}^{*}\right)$ on the same axes. If $A^{*}<A_{\mathrm{Ch}}^{*}$ at any point, the flow will be choked.

\section{Conclusion}

In this work we have developed expressions for density, temperature, pressure, and velocity profiles within arbitrarily curved ducts with arbitrarily varying cross-sectional area profiles under isentropic, compressible flow conditions where the fluid may be considered a perfect gas. These profiles are given by Equations (30), (31), (32), and (34) respectively. We have verified our results through comparison with equivalent CFD simulations. These derivations verify the assumption that is frequently made in turbomachinery texts: rothalpy is conserved along curved passages when the five requirements indicated by Lyman

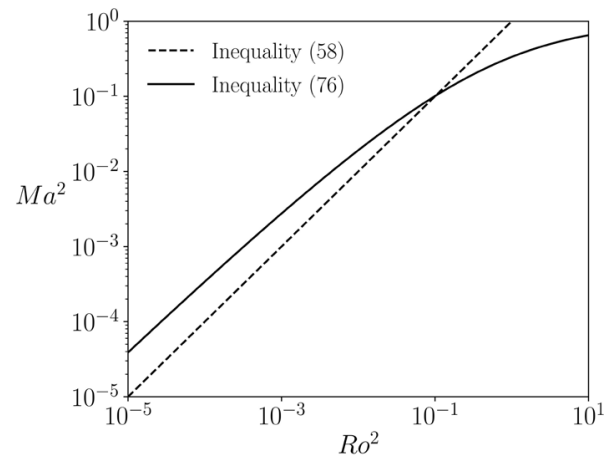

Figure 6. Restrictions on Rossby and Mach numbers dictated by the choked flow constraint for a straight, constant cross-section duct which crosses (or terminates at) the axis of rotation. All equations were evaluated with $\gamma=1.4$.

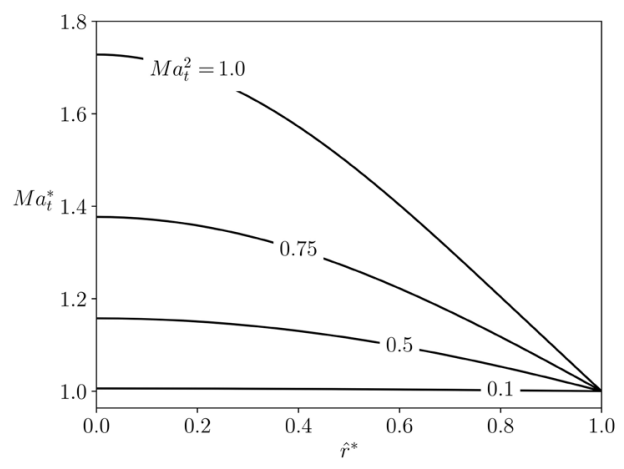

Figure 7. Plots of Equation (78) for a range of $M a_{t}^{*}$ (tip Mach numbers) with $\gamma=1.4$. 
et al. [14] are met. In addition, we have characterized the choked flow condition for compressible flow within straight ducts, clearly indicating the constraints on the choice of dimensionless groups $M a, R o, M a_{t}$, and $\gamma$ required to avoid the choked flow condition. We have characterized the variation in the critical cross-sectional area and shown how it can be used to quickly evaluate whether or not flow will choke in a rotating duct of known geometry. During this process we have identified the importance of the stagnation temperature, which may be much more pertinent than the often-used total temperature for studies involving rotating compressible flows.

\section{Acknowledgements}

The authors gratefully acknowledge the financial support from the Natural Sciences and Engineering Research Council of Canada (NSERC).

\section{Conflicts of Interest}

The authors declare no conflicts of interest regarding the publication of this paper.

\section{References}

[1] Polihronov, J.G. and Straatman, A.G. (2012) Thermodynamics of Angular Propulsion in Fluids. Physical Review Letters, 109, Article ID: 54504. https://doi.org/10.1103/PhysRevLett.109.054504

[2] Polihronov, J.G. and Straatman, A.G. (2014) Angular Propulsion the Rotational Analog of Rocket Motion. Canadian Journal of Physics, 92, 89-92.

[3] Ranque, G.J. (1933) Experiments on Expansion in a Vortex with Simultaneous Exhaust of Hot Air and Cold Air. Journal de Physique et Le Radium, 4, 112-114.

[4] Thakare, H.R., Monde, A. and Parekh, A.D. (2015) Experimental, Computational and Optimization Studies of Temperature Separation and Flow Physics of Vortex Tube: A Review. Renewable and Sustainable Energy Reviews, 52, 1043-1071. https://doi.org/10.1016/j.rser.2015.07.198

[5] Greenspan, H.P. (1968) The Theory of Rotating Fluids. CUP Archive, New York, NY, USA.

[6] Vanyo, J.P. (2015) Rotating Fluids in Engineering and Science. Elsevier, Mineola.

[7] Dixon, S.L. and Hall, C. (2013) Fluid Mechanics and Thermodynamics of Turbomachinery. 7th Edition, Butterworth-Heinemann, Oxford.

[8] Greitzer, E.M. and Tan, C.S. and Graf, M.B. (2007) Internal Flow: Concepts and Applications, Volume 3. Cambridge University Press, Cambridge.

[9] Tatro, P.R. and Mollo-Christensen, E.L. (1967) Experiments on Ekman Layer Instability. Journal of Fluid Mechanics, 28, 531-543. https://doi.org/10.1017/S0022112067002289

[10] Kristoffersen, R. and Andersson, H.I. (1993) Direct Simulations of Low-Reynolds-Number Turbulent Flow in a Rotating Channel. Journal of Fluid Mechanics, 256, 163. https://doi.org/10.1017/S0022112093002757

[11] Kheshgi, H.S. and Scriven, L.E. (1985) Viscous Flow through a Rotating Square Channel. Physics of Fluids, 28, 2968-2979. https://doi.org/10.1063/1.865136 
[12] Lieblein, S. (1952) Theoretical and Experimental Analysis of One-Dimensional Compressible Flow in a Rotating Radial-Inlet Impeller Channel. Technical Report.

[13] Cumpsty, N.A. (1989) Compressor Aerodynamics. Longman Scientific \& Technical, New York.

[14] Lyman, F.A. (1993) On the Conservation of Rothalpy in Turbomachines. Journal of Turbomachinery, 115, 520-525. https://doi.org/10.1115/1.2929282

[15] Bosman, C. and Jadayel, O.C. (1996) A Quantified Study of Rothalpy Conservation in Turbomachines. International Journal of Heat and Fluid Flow, 17, 410-417. https://doi.org/10.1016/0142-727X(96)00030-6

[16] Combrinck, M.L. and Dala, L.N. (2014) Eulerian Derivations of Non-Inertial Navier-Stokes Equations. 11th International Conference on Heat Transfer, Fluid Mechanics and Thermodynamics, Kruger National Park, 20-23 July 2015, 1-20.

[17] Kageyama, A. and Hyodo, M. (2006) Eulerian Derivation of the Coriolis Force. Geochemistry, Geophysics, Geosystems, 7, 1-5. https://doi.org/10.1029/2005GC001132

[18] Masatsuka, K. (2013) I Do Like CFD, Volume 1. 2nd Edition, Cradle.

[19] ANSYS Inc. (2011) ANSYS CFX-Pre User's Guide.

[20] Polihronov, J and Straatman, A.G. (2013) Mechanism for enhanced Energy Extraction and Cooling Pressurized Gas. WO 2013/177706 A1.

[21] Cengel, Y.A. and Boles, M.A. (2006) Thermodynamics: An Engineering Approach. 8th Edition, McGraw-Hill Higher Education, New York. 


\section{Nomenclature}

\section{Roman Symbols}

$A$ Duct or passage cross-sectional area

a Independent parameter

$A_{\mathrm{Ch}}^{*}=A_{r}^{*} / A_{t}^{*} \quad$ Critical cross-sectional area profile

$A_{t}^{*}=A / A_{t}$ Area to throat area ratio in a stationary passage

$C, D, E$ Constants of integration

$c_{p}$ Isobaric heat capacity

$c_{V}$ Volumetric heat capacity

$\dot{E}_{s t}$ Transient energy storage in a control volume

$h=\varepsilon+p / \rho$ Specific enthalpy

$i=\sqrt{-1}$

$\hat{i}, \hat{j}, \hat{k}$ Unit vectors aligned with the $\mathrm{x} ; \mathrm{y}$; and $\mathrm{z}$ axes, respectively

$\dot{m}$ Mass ow rate

$\dot{m}_{C h}$ Maximum (choked) mass ow rate

$\dot{m}_{C h \text {,min }}$ Minimum choked mass ow rate; occurs at the location in a duct or passage which will choke first if the mass ow rate is slowly increased.

$p$ Thermodynamic pressure

$\mathrm{P}^{*}$ Parameterized position vector

$R_{8}$ Specific ideal gas constant

$T_{0}=T+\frac{u^{2}}{2 c_{p}}$ Total temperature

$\mathrm{t}$ Unit tangent vector to a parametric curve $\mathrm{P}^{*}$

u Velocity

$\bar{u}$ Flow speed along along a constrained path

$\mathrm{x}$ Position vector

$X, Y, Z$ Components of position vector

$X, y, Z$ Cartesian co-ordinates

\section{Greek Symbols}

$\gamma=\frac{c_{p}}{c_{v}}$ Ratio of speci_c heats

$\nabla$ Gradient operator

$\varepsilon$ speci_c internal energy

$\rho$ fluid density

$\phi$ Arbitrary scalar or vector quantity

$\bar{\phi} 0$ Stagnation quantity

$\Omega$ Angular velocity of rotating frame

\section{Dimensionless Groups}

$M a^{2}=\frac{u_{c}^{2}}{\gamma R_{s} T_{c}}$ Global Mach number

$M a_{\mathrm{S}}=\frac{u_{2}}{\sqrt{\gamma R_{s} T_{2}}}$ Shroud Mach number 
$M a_{L}=\frac{\hat{u}}{\sqrt{\gamma R_{s} T}}$ Local Mach number

$M a_{t}=\frac{\Omega_{c} x_{c}}{\sqrt{\gamma R_{s} \bar{T}_{0}}}$ Tip Mach number

$M a_{t} *$ Modified tip Mach number ratio

$R o=\frac{u_{c}}{\Omega_{c} x_{c}}$ Rossby number

\section{Superscripts}

* Non-dimensional quantity

\section{Subscripts}

1 Quantity at boundary nearest to the center of rotation

2 Quantity at boundary furthest from the center of rotation

c Characteristic dimension

in Quantity entering a control volume

out Quantity exiting a control volume

Other Symbols

' Derivative of single-variable function

$\wedge$ Quantity in non-stationary frame

\section{Acronyms}

1D One-dimensional

CFD Computational Fluid Dynamics

RHVT Ranque-Hilsch Vortex Tube

RHS Right Hand Side 